\title{
LIGAMENT AND MENISCAL INJURIES FOLLOWING KNEE TRAUMA- ROLE OF THE MRI AND ITS CORRELATION WITH ARTHROSCOPY
}

\author{
Ambika Sumeet Juwarkar ${ }^{1}$, Nilkanth Laxman Pal ${ }^{2}$ \\ ${ }^{1}$ Assistant Professor, Department of Radiodiagnosis, Goa Medical College, Bambolim, Goa. \\ ${ }^{2}$ Senior Resident, Department of Radiodiagnosis, Goa Medical College, Bambolim, Goa.
}

\section{ABSTRACT}

\section{BACKGROUND}

MRI is an excellent imaging modality for assessing internal derangement of the knee. Its non-invasive nature, excellent soft tissue contrast and no radiation risk makes MRI a boon in knee imaging.

The aims of the study are-

1. To know the occurrence of meniscal and ligament injuries following knee injury.

2. To evaluate diagnostic performance of MRI following knee injury in correlation with arthroscopy.

\section{MATERIALS AND METHODS}

This is a prospective study including 70 patients conducted at our institution over a period of 3 years from October 2006 to October 2009. Only the patients with history of knee trauma were included in our study. All the patients initially underwent MRI and subsequent arthroscopy. The pattern of injury was recorded. The location, type and grades of meniscal and ligament tears were recorded by experienced radiologist. Later MRI findings were correlated with arthroscopy findings.

\section{RESULTS}

On evaluation of MRI findings of 70 patients including 60 (85.7\%) male and $10(14.28 \%)$ female patients, the most commonly detected injuries were of anterior cruciate ligament. The sensitivity, specificity and accuracy of MRI for detecting medial meniscal injury were $92.3 \%, 90.9 \%$ and $91.4 \%$ respectively. Lateral meniscus $(88.0 \%, 88.9 \%$ and $88.6 \%$ respectively). Anterior cruciate ligament (91.8\%, 95.2\% and $92.9 \%$ respectively) and posterior cruciate ligament (100\%, 98.5\% and 98.6\% respectively). Comparisons between qualitative variables were done using the chi-square test.

\section{CONCLUSION}

MRI is highly sensitive and accurate at identification of cruciate ligament and meniscal tears. A close agreement was obtained between MRI and arthroscopic diagnosis. In case of meniscal tears, full description of meniscal tear (location, grade and configuration) had important implications on treatment of these patients.

\section{KEYWORDS}

Anterior Cruciate Ligament (ACL), Posterior Cruciate Ligament (PCL), Medial Meniscus, Lateral Meniscus, Knee Magnetic Resonance Imaging (MRI), Arthroscopic Correlation, Diagnostic Performance.

HOW TO CITE THIS ARTICLE: Juwarkar AS, Pal NL. Ligament and meniscal injuries following knee trauma- role of the MRI and its correlation with arthroscopy. J. Evolution Med. Dent. Sci. 2017;6(81):5733-5738, DOI: 10.14260/jemds/2017/1243

\begin{abstract}
BACKGROUND
MRI is an excellent imaging modality for assessing internal derangement of the knee. Its non-invasive nature, excellent soft tissue contrast and no radiation risk makes MRI a boon in knee imaging. [1-3] Plain $\mathrm{x}$-rays and computed tomography can suggest osseous problems, not the soft tissue injuries. Arthroscopy is currently considered gold standard for evaluating injuries to menisci and ligaments. Undoubtedly, it is an invasive procedure with possible complications like infection, nerve injuries, vascular injuries and even pulmonary embolism. ${ }^{[4]}$ Thus, MRI is a good screening tool secondary to its higher negative predictive value and higher specificity avoiding unnecessary arthroscopies.[5] As already
\end{abstract}

'Financial or Other Competing Interest': None.

Submission 21-08-2017, Peer Review 28-09-2017,

Acceptance 04-10-2017, Published 09-10-2017.

Corresponding Author:

Dr. Ambika Sumeet Juwarkar,

Jeet Bldg, $3^{\text {rd }}$ Floor,

Airport Road,

Chicalim-Goa.

E-mail: ambika_juwarkar@yahoo.com nil25.pal@gmail.com

DOI: $10.14260 /$ jemds $/ 2017 / 1243$ known, MRI can provide far more information about ligament and meniscal injuries than all other radiological imaging combined together. Despite these prospects, accuracy of MRI in imaging of meniscal and ligament tears has been challenged a number of times.

MRI is considered to have good efficacy in providing anatomical and pathological details of knee with good sensitivity, specificity and accuracy as reported in many previous studies.[6-10] Although experienced surgeon can determine the presence of meniscal and ligament injuries through physical examination, MRI is recommended to avoid unrequired arthroscopies which results from false positive physical tests, especially in presence of multiple injuries.

Hence, this study is undertaken to analyse diagnostic performance of the MRI in determining meniscal and ligament injuries in local population in comparison with arthroscopy regarding it as the gold standard.

\section{MATERIALS AND METHODS}

This is a descriptive study including 70 patients conducted at our institution over a period of 3 years from October 2006 to October 2009.

Concurrence was taken from the chairman, academic committee, scientific committee and ethical committee for 
this study. Informed consent was taken from patients.

Patients between 15 - 35 years of age group with recent history of knee trauma were included in the study. Patients below 15 years and patient above 35 years were excluded, because this population group can show abnormal meniscal and ligament signal without tear secondary to vascularity and degenerative changes in respective age groups. Patients who had previously undergone arthroscopy were excluded, because arthroscopic repair caused distortion of the normal anatomy of the menisci and ligaments which cannot be attributed to trauma. No sex preference was introduced in the study.

Time interval between occurrence of injury and date of scanning varied from 5 days to 1 year and between the date of MR imaging and arthroscopy varied according to the convenience of the patient, surgeon and availability of operating room.

\section{Imaging Technique}

MR imaging was performed at 1.5 Tesla MRI machine (Siemens Magnetom Avanto) at our institution. Imaging of menisci and ligaments were performed in all three standard planes (sagittal, coronal and axial). The field of view between 14 - $16 \mathrm{cms}$, matrix 192 - 256 and slice thickness was $3 \mathrm{~mm}$.

The standard imaging protocol included axial and sagittal images T2 weighted images $(\mathrm{TR} / \mathrm{TE}=3580 / 88)$ without fat saturation, sagittal proton density image $(\mathrm{TR} / \mathrm{TE}=3580 / 15)$ without fat saturation, coronal T2-weighted fat saturated image $(\mathrm{TR} / \mathrm{TE}=5480 / 31)$ and $3-\mathrm{D}$ gradient echo sequence (for cartilage).

\section{Interpretation of Images}

MR images were reviewed by 2 senior radiologists in our department. Meniscal tears were evaluated for their site (anterior horn, body and posterior horn), plane (inner $1 / 3^{\text {rd }}$ and outer $2 / 3^{\text {rd}}$ ), grade and configuration (horizontal, longitudinal, radial, root, complex, bucket-handle tears and displaced). ACL and PCL tears and the site of tears (femoral, tibial attachment and mid-substance) were also recorded.

Lack of an intrameniscal high signal and normal morphology was considered as a normal meniscus. Presence of intrameniscal high signal reaching upto the articular surface was considered as tear, whereas those signal intensities not reaching articular surface were not labelled as tears. The ACL was considered normal when it appeared as a band of fibres of low or intermediate signal intensity on both sagittal and coronal images. Fuzzy ligament with ill-defined outline and abnormal signal intensity was considered partial tear and completely torn ACL shows complete disruption of all fibres, discontinuity or avulsion from its attachments.

\section{Arthroscopy}

The results of MR imaging were reported to the orthopaedic surgeon. Prior knowledge of the complete description of the tear helped surgeons to use second portal for optimal visualisation and probing of the area containing abnormal signal. All Arthroscopies were done in our hospital environment with complete preoperative care by experienced surgeons. Standard portals were used for entry. Data was recorded.

Collateral ligaments and cartilage injuries were not included in this study.

\section{Statistics}

Collected data was analysed using SPSS 13 computer program. Comparisons between qualitative variables were done using the chi-square test. $\mathrm{P}$ values $<0.05$ were considered as statistically significant. Sensitivity, specificity, positive and negative predictive values, accuracy and $P$ value were calculated to test validity of MRI against arthroscopy considering latter as gold standard.

\section{RESULTS}

Total 70 patients were included in this study involving 60 (85.7\%) male and $10(14.28 \%)$ female patients; 46 patients (65.71\%) had derangement of right knee and 24 (34.28\%) had derangement of left knee.

Diagnostic performance of MRI is shown in Table No. 1.

\begin{tabular}{|c|c|c|c|c|c|}
\hline & Sensitivity & Specificity & PPV & NPV & Accuracy \\
\hline $\begin{array}{c}\text { Medial } \\
\text { meniscus }\end{array}$ & $92.3 \%$ & $90.9 \%$ & $85.7 \%$ & $95.2 \%$ & $91.4 \%$ \\
\hline $\begin{array}{c}\text { Lateral } \\
\text { meniscus }\end{array}$ & $88.0 \%$ & $88.9 \%$ & $81.5 \%$ & $93.0 \%$ & $88.6 \%$ \\
\hline ACL & $91.8 \%$ & $95.2 \%$ & $97.8 \%$ & $88.3 \%$ & $92.9 \%$ \\
\hline PCL & $100 \%$ & $98.5 \%$ & $80.0 \%$ & $100 \%$ & $98.6 \%$ \\
\hline Table 1. Validity of MRI in Correlation with Arthroscopy \\
\hline
\end{tabular}

(PPV= Positive predictive value, NPV= Negative predictive value, $\mathrm{ACL}=$ Anterior cruciate ligament, $\mathrm{PCL}=$ Posterior cruciate ligament).

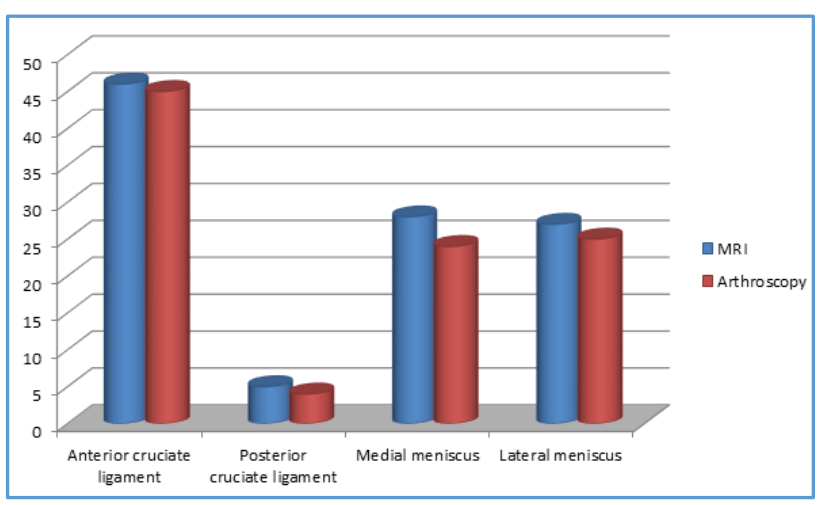

Figure 1. Graph showing Number of Various Tears on MRI and Arthroscopy

Various tears detected in our study are placed in Figure No. 2.

The most common pathology detected on MRI as well as on arthroscopy was the anterior cruciate ligament tear and the least common was posterior cruciate ligament tear.

Meniscal Tears (Figures 3, 4, 5, 6 and 7)

Out of the 70 cases in this study, 28 patients (40\%) had medial meniscus and 27 patients (38.57\%) had lateral meniscus tears on MRI. 


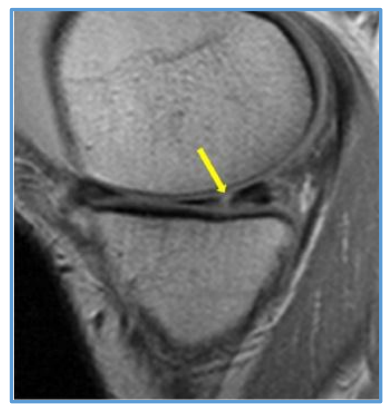

Figure 2a

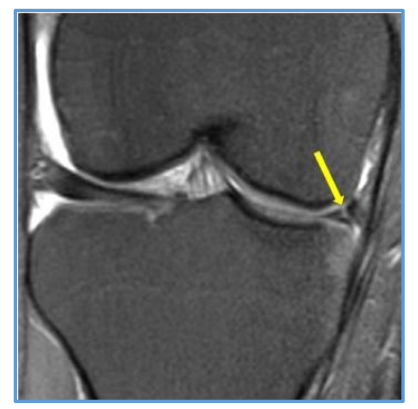

Figure $2 b$
Figure 2. Longitudinal Vertical Tear. Figure 2a. (Proton Density Sagittal Image) shows the Body of the Lateral Meniscus with a High Signal Intensity Vertical Cleft (Arrow) extending to both the Articular Surfaces s/o Vertical Tear. Figure $2 b$. (Coronal T2 Weighted Image with Fat Saturation) confirms the Longitudinal Nature of this Tear (Arrow).

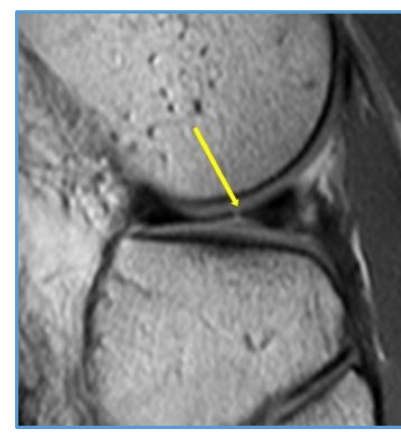

Figure 3a

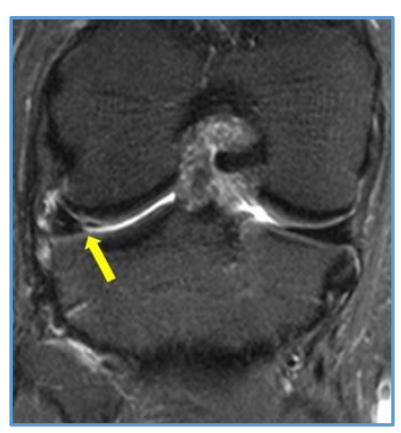

Figure $3 b$
Figure 3. Radial Tear. Figure 3a. (T2-Weighted Sagittal Image) shows Body of Lateral Meniscus with a High Signal Intensity Cleft extending to both the Articular Surfaces (Cleft Sign) shown by Long Arrow. This Cleft produced Truncated appearance of Inner Part of the Body of the Lateral Meniscus (Truncated Triangle Sign) suggestive of a Radial Tear shown by Arrow in Figure 3b. (Coronal T2 Weighted Image with Fat Saturation). The Medial Meniscus has the normal Triangular Configuration.

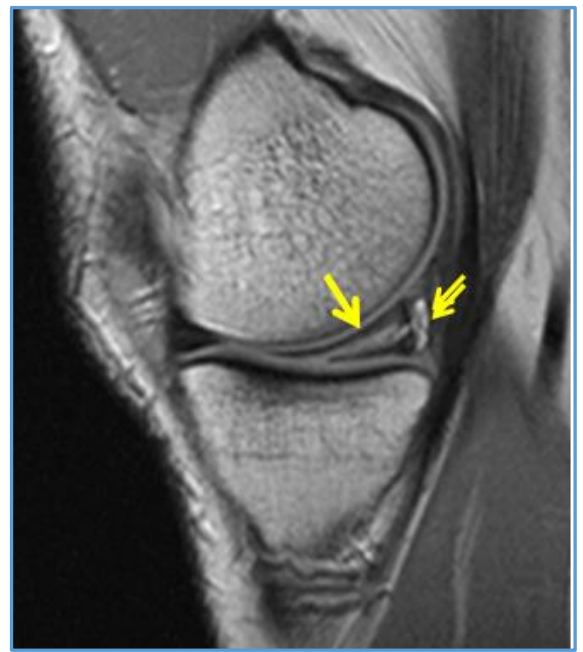

Figure 4. Horizontal Oblique Tear. Sagittal T2 Weighted Image shows Horizontal Oblique Tear (Arrow) reaching upto the Inferior Articular Surface associated with Parameniscal Cyst (Double Arrow)

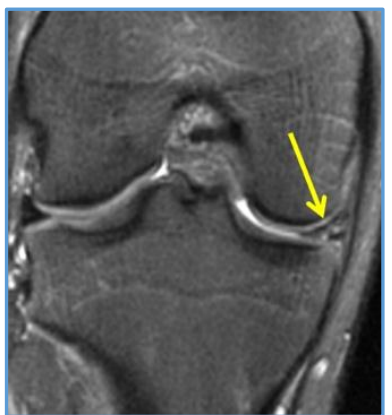

Figure 5a

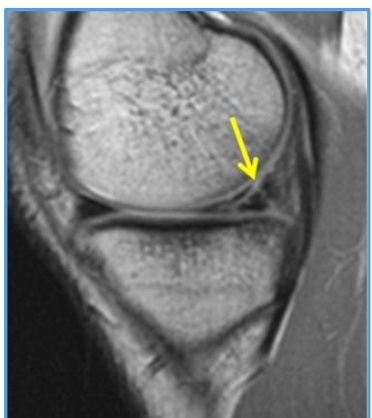

Figure 5b
Figure 5. Complex Meniscal Tear. Figure 5a. (Sagittal Proton Density Image) and Figure 5b. (Coronal T2 Weighted Image with Fat Saturation) shows a Stellate Shaped Hyperintense Signal in the Posterior Horn of Medial Meniscus (Arrows) having both Longitudinal and Horizontal Component suggestive of Complex Tear.

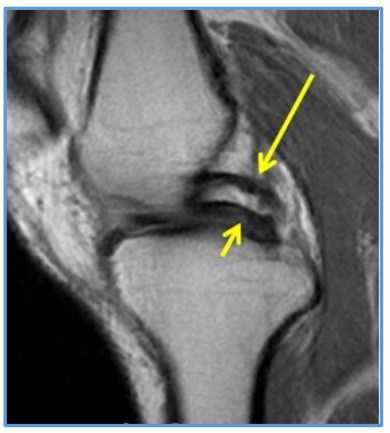

Figure 6a

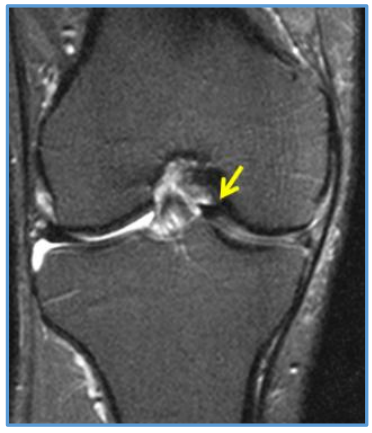

Figure $6 b$
Figure 6. Bucket Handle Tear. Figure 6a. (Sagittal T2 Weighted Image) shows Double PCL Sign formed by Flipped Inner Fragment of Torn Meniscus (Short Arrow) located Anterior to Posterior Cruciate Ligament (Long Arrow). Figure 6b. (Coronal T2 Weighted Image with Fat Saturation) shows Flipped Meniscal Fragment in the Intercondylar Region (Arrow).
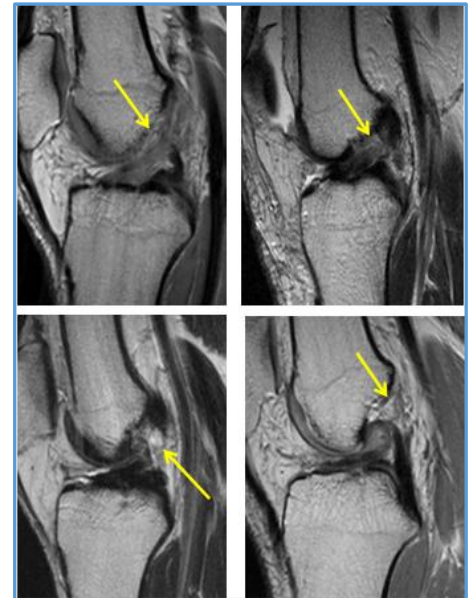

Figure 7. Tears of the Anterior Cruciate Ligament. Figure 7a. (Sagittal Proton Density Image) shows Bulky ACL (Arrow) having Altered Signal Intensity with Intact Fibres s/o Partial Tear. Figure 7b. (T2-Weighted Sagittal Images) show High-Grade Partial Tear with > 75\% Fibres Torn (Arrow). Figure 7c. (T2-Weighted Sagittal Image) shows complete Midsubstance Tear (Arrow). Figure 7d. (Proton Density Sagittal Image) shows Complete Tear of ACL from the Femoral Attachment (Arrow). 


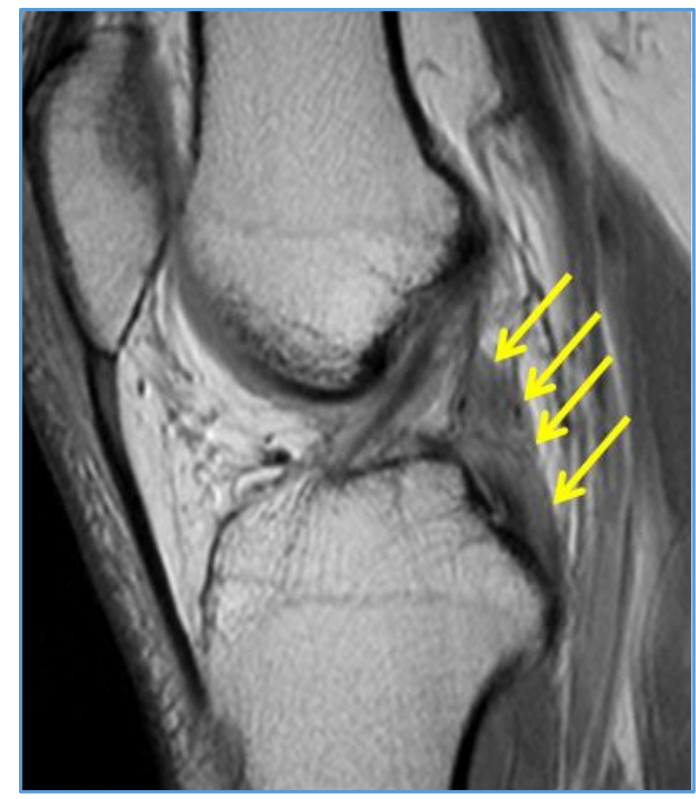

Figure 8. Sagittal Proton Density image shows Hyperintense Signal in the Posterior Cruciate Ligament (Arrows) with Intact Fibres s/o Partial Tear

\section{Medial Meniscus}

Out of total 28 medial meniscus tears identified on MRI, only $24(34.28 \%)$ tears were confirmed on arthroscopy.

On MRI, the most common site medial meniscal tear was the posterior horn seen in 17 cases (54.8\%). Although, the most common site of tear on arthroscopy was also the posterior horn 14 (45.16\%), three tears which were identified on MRI were not visualised on arthroscopy.

There were 13 longitudinal tears identified on MRI, whereas $8(35 \%)$ were identified on arthroscopy. There were $5(19 \%)$ bucket handle tears and $3(11 \%)$ horizontal tears, which were identified on MRI and all of these tears were confirmed on arthroscopy.

There were 3 radial tears identified on MRI, but only 2 were found on arthroscopy. There were 4 complex tears seen on MRI, but arthroscopy could identify only 3.

\section{Lateral Meniscus}

There were $27(38.75 \%)$ tears of the lateral meniscus identified on MRI and 25 (35.71\%) tears were found on arthroscopy.

The most common site of tear on MRI as well as arthroscopy was body of lateral meniscus.

The most common type of tear of lateral meniscus on MRI was radial tear seen in 12 patients. On arthroscopy, most common type of tear was also radial tear. Two radial tears, which were found on arthroscopy were not identified on MR imaging. There were 2 horizontal and 5 longitudinal tears identified on both MRI and arthroscopy. There were 5 bucket handle tears on MRI, but only 3 were seen on arthroscopy. Similarly, 3 complex tears were seen on MRI, but only 1 was found on arthroscopy.

\section{Cruciate Ligament Tears (Figure 8 and Figure 9)}

Out of the 70 cases studied, 46 patients (65.71\%) had ACL tear and 5 patients $(7.1 \%)$ had PCL tears on MRI.
45 out of the 46 tears identified on MRI were identified on arthroscopy. The most common site of ACL tear was at its femoral attachment on MRI as well as on arthroscopy.

\section{Posterior Cruciate Ligament}

There were 5 patients of PCL tear identified on MRI and 4 were confirmed on arthroscopy. Most common type of tear was the mid-substance tear.

\section{DISCUSSION}

\section{Medial Meniscus}

The sensitivity, specificity and accuracy of identifying medial meniscus tear by MRI in our study were $92.3 \%, 90.9 \%$ and $91.4 \%$ respectively. This findings correlate well with the previous studies.[6-10]

In our study, the most common site of tear was the posterior horn. The posterior horn of medial meniscus was also reported as the commonest site of tear by Stoller et al[11] in their study. They attributed this to the fact that increased injury is generated from anatomic and mechanical loading forces generated from this location.

In our study, the most common site of tear on arthroscopy was also the posterior horn. Three tears which were identified on MRI were not visualised on arthroscopy. This discrepancy was also reported in previous studies such as of that of Lawrence et al[12]; however, they said that degenerative changes in the menisci in elderly population and increased meniscal vascularity in young patient are caused for false positive MRI signals, which are not perceived on arthroscopy as tear. Since we had excluded these age groups from our study, we had to search for alternative answer for this discrepancy. Carrino et al[13] found that posterior horn of meniscus is a hidden corner of arthroscopy, especially the area between the medial collateral ligament and the intercondylar attachment of the posterior horn, thus is frequently missed on arthroscopy. They suggested multiple puncture during arthroscopy will reduce such negative arthroscopic examinations.

The most common type of medial meniscal tear identified on MRI and arthroscopy was longitudinal tear. Some of the longitudinal tears identified on MRI were not seen on arthroscopy. Possible explanation for this was given by De Smet et al [14] in their study, wherein they stated longitudinal tears of the medial meniscus which were identified on MRI had actually healed and was therefore not confirmed on arthroscopy. This hypothesis is also supported by Weis et $\mathrm{al}^{[15]}$ in their study. They attributed this to the fact that most of the longitudinal tears were present in the outer $1 / 3^{\text {rd }}$ of the meniscus, which has a rich vascular supply and good potential for healing.

\section{Lateral Meniscus}

We recorded high sensitivity, specificity and accuracy of identification of the lateral meniscus tear by MRI correlating well with previous studies.[8-10] The recorded percentages were $88.0 \%, 88.9 \%$ and $88.6 \%$ respectively.

The most common site of lateral meniscal tears on MRI as well as on arthroscopy in our study was the body of the lateral meniscus. This is in contrary to the study by Singh et $\mathrm{al}^{\left[{ }^{[9]}\right.}$ where the most common site of lateral meniscal tear was posterior horn of lateral meniscus.

\section{Anterior Cruciate Ligament}


In our study, tears of the anterior horn of lateral meniscus were low on MRI as well as on arthroscopy. This correlate well with the study done by Shankman et al,[16] wherein they stated that tear of the anterior horns of lateral meniscus is unusual and false positive findings on MRI are probably due to increased signal in the anterior horn of lateral meniscus, because of the junction in the fibrocartilage and collagen at the central attachment site of the anterior horn of lateral meniscus and anterior cruciate ligament. This signal intensity changes can be mistaken as tear.

The most common type of tear of lateral meniscus on MRI was radial tear. On arthroscopy also, the most common type of tear was radial tear.

Treatment of the meniscal tears is dependent on their configuration, size and location. Longitudinal and oblique tears are usually amenable to repair, whereas radial tears, horizontal tears and complex tears (of which there is frequently a radial component) generally cannot be repaired and usually require partial meniscectomy. Thus, characterisation of the tear can help surgeon and patients understand the preoperative likelihood of repair versus resection. This aids in preoperative planning, patient counselling and rehabilitation planning.

Based on our study, we realised that evaluation of menisci on both sagittal and coronal planes is necessary to improve MRI efficacy. We also realised that T2* weighted GRE images better showed the meniscal tears than the FSE images.[17]

\section{Anterior Cruciate Ligament}

In our study the sensitivity, specificity and accuracy of MRI to identify ACL tear were $91.8 \%, 95.2 \%$ and $92.9 \%$ respectively. This correlates well with various previous studies in literature.[6,7,9,10,18,19]

Discordant appearance of ACL (when one MR sequence shows disrupted or poorly seen ACL fibres and other sequences show intact ACL fibres) and partial ACL tears were not reported in this study, because previous studies such as Smith et $\mathrm{al}^{[20]}$ and Umans et al[21] have proved that in these cases ACL was normal when seen on arthroscopy. Thus, overall accuracy of the MRI in our study improved. Also, it is important to know that only complete ACL tears needs reconstruction, whereas partial ACL tear can be treated with physical rehabilitation.

The most common site of ACL tear on MRI was its femoral attachment on MRI as well as on arthroscopy. This was in contrary to the study by Berquist et al,[22] where midsubstance tear was the commonest.

Mink et al[19] found that ACL tear at the femoral attachment can be missed due to the partial volume averaging of the proximal ligament with the sagittal oriented medial face of the lateral femoral condyle. In our study, we did encounter such problem; however, in such situations we examined ACL in other planes like axial and coronal to solve the issue. Therefore, we recommend evaluating ACL in all three planes to avoid false result.

\section{Posterior Cruciate Ligament}

The sensitivity, specificity and accuracy of MRI identifying PCL tear were $100 \%, 98.5 \%$ and $98.6 \%$ respectively. Posterior cruciate ligament injuries are less common than ACL injuries.
Most common type of posterior cruciate ligament tear was the mid-substance tear. This finding has also emerged in the earlier studies by Roberts et al,[23] Sonin et al[24] and Grover et al.[25] Most of these studies agree that sagittal images are best for diagnosis of mid-substance tear.

Rubin et al[26] suggested that in presence of multiple injuries affects sensitivity and specificity of MRI. According to results of our study, we deviate from such postulations. Although, the diagnostic performance of MRI decreases in patients with multiple injuries, we did not find statistical significance supporting this finding. Increased laxity of tissue due to multiple injuries allows adequate arthroscopic evaluation of all areas increasing the detection rate of arthroscopy. Secondly extensive oedema/ haemorrhage and altered anatomical relationship due to multiple injuries further decrease the accuracy of MRI.

\section{Limitations}

The design of our study had certain limitations. Arthroscopy is not truly a gold standard for detecting all soft tissue injuries of the knee. Surgeons were aware of the MRI findings before arthroscopy, thus bias was introduced in the study.

\section{CONCLUSION}

Our study thus proves that diagnostic performance of MRI is excellent with high sensitivity, specificity and accuracy for meniscal and ligament injuries of the knee joint. The accuracy of MRI allows it to be a good screening tool for internal derangement of the knee. It helps targeting arthroscopy to those patients who are likely to obtain therapeutic benefits avoiding an invasive procedure in other patients. In addition, MRI can help surgeon to plan therapeutic arthroscopy in case of meniscal and ligament injuries, thus improving patient's outcome.

\section{REFERENCES}

[1] Morrison WB, Sanders TG. Imaging of the knee. Problem solving in musculoskeletal imaging. Philadelphia, PA: Mosby Elsevier 2008:567-8.

[2] Glashow JL, Katz R, Schneider M, et al. Double-blind assessment of the value of MRI in the diagnosis of ACL and meniscal lesions. J Bone Joint Surg Am 1989;71(1):113-9.

[3] Ben-Galim P, Steinberg EL, Amir H, et al. Accuracy of magnetic resonance imaging of the knee and unjustified surgery. Clin Orthop Relat Res 2006;447:100-4.

[4] Small NC. Complications in arthroscopic meniscal surgery. Clin Sports Medicine 1990;9(3):609-17.

[5] Elvenes J, Jerome CP, Reikeras 0, et al. MRI as a screening procedure to avoid arthroscopy for meniscal tears. Arch Orthop Trauma Surg 2000;120(1-2):14-6.

[6] Mackenzie R, Dixon AK, Keene GS, et al. Magnetic resonance imaging of the knee: assessment of effectiveness. Clin Radiol 1996;51(4):245-50.

[7] Oei EH, Nikken JJ, Verstijnen AC, et al. MR imaging of the menisci and cruciate ligaments: a systemic review. Radiology 2003;226(3):837-48. 
[8] Cheung LP, Li KC, Hollett MD, et al. Meniscal tears of the knee: accuracy of detection with fast spin-echo MR imaging and arthroscopic correlation in 293 patients. Radiology 1997;203(2):508-12.

[9] Singh JP, Garg L, Shrimati R, et al. MR imaging of knee with arthroscopic correlation in twisting injuries. Indian J Radiol Imaging 2004;14(1):33-40.

[10] Polly DW, Callaghan JJ, Sikes RA, et al. The accuracy of selective magnetic resonance imaging compared with the findings of arthroscopy of the knee. J Bone Joint Surg Am 1988;70(2):192-8.

[11] Stoller DW, Martin C, Crues JV, et al. Meniscal tears: pathological correlation with MR imaging. Radiology 1987;163(3):731-5.

[12] Burk DL, Mitchell DG, Rifkin MD, et al. Recent advances in MRI of the knee. Radiol Clin of North America 1990;28(2):379-93.

[13] Carrino JA, Schweitzer ME. Imaging of sports-related knee injuries. Radiol Clin of North America 2002;40(2):181-202.

[14] De Smet AA, Graf BK. Meniscal tears missed on MR imaging: relationship to meniscal tear patterns and anterior cruciate ligament tears. Am J Roentgenol 1994;162(4):905-11.

[15] Weiss KL, Morehouse HT, Levy IM. Sagittal MR images of the knee: a low-signal band parallel to the posterior cruciate ligament caused by a displaced bucket-handle tear. Am J Roentgenol 1991;156(1):117-9.

[16] Shankman S, Beltran J, Melamed E, et al. Anterior horn of the lateral meniscus: another potential pitfall in MR imaging of the knee. Radiology 1997;204(1):181-4.

[17] Rubin DA, Kneeland JB, Listerud J, et al. MR diagnosis of meniscal tears of the knee: value of FSE vs conventional SE pulse sequences. Am J Roentgenol 1994;162(5):1131-5.
[18] Ha TP, Li KC, Beaulieu CF, et al. Anterior cruciate ligament injury: fast spin-echo MR imaging with arthroscopic correlation in 217 examinations. Am J Roentgenol 1998;170(5):1215-9.

[19] Mink JH, Levy T, Crues JV. Tears of the anterior cruciate ligament and menisci of the knee: MR imaging evaluation. Radiology 1988;167(3):769-74.

[20] Smith DK, May DA, Phillips P. MR imaging of the anterior cruciate ligament: frequency of discordant findings on sagittal-oblique images and correlation with arthroscopic findings. Am J Roentgenol 1996;166(2):411-3.

[21] Umans H, Wimpfheimer 0 , Haramati $N$, et al. Diagnosis of partial tears of the anterior cruciate ligament of the knee: value of MR imaging. Am J Roentgenol 1995;165(4):893-7.

[22] Berquist TH. Magnetic resonance techniques in musculoskeletal diseases. Rheum Clin North Am 1991;17(3):599-615.

[23] Roberts CC, Towers JD, Spangehl MJ, et al. Advanced MR imaging of the cruciate ligaments. Magn Reson Imaging Clin N Am 2007;15(1):73-86.

[24] Sonin AH, Fitzgerald SW, Friedman H, et al. Posterior cruciate ligament injury: MR imaging diagnosis and pattern of injury. Radiology 1994;190(2):455-8.

[25] Grover JS, Bassett LW, gross ML, et al. Posterior cruciate ligament: MR imaging. Radiology 1990;174(2):527-30.

[26] Rubin DA, Kettering JM, Towers JD, et al. MR imaging of knees having isolated and combined ligament injuries. Am J Roentgenol 1998;170(5):1207-13. 